\title{
A Simple Proof of Sharkovsky's Theorem
}

\author{
Bau-Sen Du \\ Institute of Mathematics \\ Academia Sinica \\ Taipei 11529, Taiwan \\ dubs@math.sinica.edu.tw
}

\begin{abstract}
In this note, we present a simple directed graph proof of Sharkovsky's theorem.
\end{abstract}

\section{Introduction.}

Throughout this note, $I$ is a compact interval, and $f: I \rightarrow I$ is a continuous map. For each integer $n \geq 1$, let $f^{n}$ be defined by: $f^{1}=f$ and $f^{n}=f \circ f^{n-1}$ when $n \geq 2$. For $y$ in $I$, we call the set $O_{f}(y)=\left\{f^{k}(y) \mid k \geq 0\right\}$ the orbit of $y$ (under $f$ ) and call $y$ a periodic point of $f$ with least period $m$ (or a period- $m$ point of $f$ ) if $f^{m}(y)=y$ and $f^{i}(y) \neq y$ when $0<i<m$. If $f(y)=y$, then we call $y$ a fixed point of $f$. It is clear that every $f$ of the type in question has fixed points.

For discrete dynamical systems defined by iterated interval maps, one of the most remarkable results is Sharkovsky's theorem [5], [6]. It states that, if $f$ has a period- $m$ point, then $f$ also has a period- $n$ point precisely when $m \prec n$ in the following Sharkovsky's ordering :

$$
3 \prec 5 \prec 7 \prec \cdots \prec 2 \cdot 3 \prec 2 \cdot 5 \prec 2 \cdot 7 \prec \cdots \prec 2^{2} \cdot 3 \prec 2^{2} \cdot 5 \prec 2^{2} \cdot 7 \prec \cdots \prec 2^{3} \prec 2^{2} \prec 2 \prec 1 .
$$

It is well known (see [8]) that the sufficiency of Sharkovsky's theorem can be derived from the following three statements: (a) if $f$ has a periodic point of least period greater than 2 , then $f$ also has a periodic point of least period 2; (b) if $f$ has a periodic point of odd period $m \geq 3$, then $f$ also has a periodic point of least period $n$ for every integer $n$ such that $n \geq m+1$; (c) if $f$ has a periodic point of odd period $m \geq 3$, then $f$ also has periodic points of all even periods. The difficulty of proving the sufficiency of Sharkovsky's theorem lies in proving (c), where most proofs involve the structures of the so-called Štefan cycles [1], [3], [7]. In this note, we give a unified proof of (b) and (c) that does not involve Štefan cycles. We also give a different proof of (a) [2], [8]. For the sake of completeness, we end the paper with a proof of Sharkovsky's theorem.

In proving Sharkovsky's theorem, we need the following result [3, p.12]. 
Lemma 1. Let $k, m, n$, and $s$ be positive integers. Then the following statements hold:

(1) If $y$ is a periodic point of $f$ with least period $m$, then it is a periodic point of $f^{n}$ with least period $m /(m, n)$, where $(m, n)$ is the greatest common divisor of $m$ and $n$.

(2) If $y$ is a periodic point of $f^{n}$ with least period $k$, then it is a periodic point of $f$ with least period $\mathrm{kn} / \mathrm{s}$, where $s$ divides $n$ and is relatively prime to $k$.

\section{A proof of (a).}

We need the following result, which can also be used to show that $f$ has no period-2 point if and only if for each point $c$ of $I$ the iterates $f^{n}(c)$ converge to a fixed point of $f$ [3, p.121].

Lemma 2. If $c$ and $d$ are points of I such that $f(d) \leq c<d \leq f(c)$, then $f$ has a periodic point of least period 2.

Proof. Write $I=[a, b]$. Let $w=\min \{c \leq x \leq d \mid f(x)=x\}$, and let $v$ be a point in $[c, w]$ with $f(v)=d$. Then, $f^{2}(v)=f(d) \leq c \leq v$. If $f$ has no fixed point in $[a, c]$, then it fixes no point of $[a, v]$. Since $f^{2}(a) \geq a$, it follows that $f$ has a periodic point with least period 2 in $[a, v]$. If $f$ has a fixed point in $[a, c]$, let $t=\max \{a \leq x<c \mid f(x)=x\}$. Then $f$ has no fixed point in $(t, v]$. Let $u$ be a point in $[t, c]$ with $f(u)=c$. Then $f^{2}(u)=f(c) \geq d>u$. Since $f^{2}(v) \leq v$, we infer that $f^{2}(y)=y$ for some $y$ in $[u, v]$. Because $f$ has no fixed point in $[u, v], y$ is a periodic point of $f$ with least period 2 .

Proposition 3. If $f$ has a periodic point of least period $m$ larger than 2 , then $f$ also has a periodic point of least period 2.

Proof. Let $P=\left\{x_{i} \mid 1 \leq i \leq m\right\}$, with $x_{1}<x_{2}<\cdots<x_{m}$, be a period- $m$ orbit of $f$. Since $x_{1}<f\left(x_{1}\right)$ and $f\left(x_{m}\right)<x_{m}$, there exists an integer $s$ satisfying $1 \leq s \leq m-1$ such that $x_{s}=$ $\max \{x \in P \mid x<f(x)\}$. It is clear that $x_{s+1} \leq f\left(x_{s}\right)$ and $f\left(x_{s+1}\right) \leq x_{s}$. By Lemma $2, f$ has a periodic point of least period 2 .

\section{A unified proof of (b) and (c).}

If there are closed subintervals $J_{0}, J_{1}, \cdots, J_{n-1}, J_{n}$ of $I$ with $J_{n}=J_{0}$ such that $f\left(J_{i}\right) \supset J_{i+1}$ for $i=0,1, \cdots, n-1$, then we say that $J_{0} J_{1} \cdots J_{n-1} J_{0}$ is a cycle of length $n$. We require the following result.

Lemma 4. If $J_{0} J_{1} J_{2} \cdots J_{n-1} J_{0}$ is a cycle of length $n$, then there exists a periodic point $y$ of $f$ such that $f^{i}(y)$ belongs to $J_{i}$ for $i=0,1, \cdots, n-1$ and $f^{n}(y)=y$.

We now give a simple unified proof of (b) and (c). 
Proposition 5. If $f$ has a periodic point of least period $m$ with $m \geq 3$ and odd, then $f$ has periodic points of all even periods. Furthermore, $f$ has a periodic point of least period $n$ for each integer $n$ with $n \geq m+1$.

Proof. Let $P=\left\{x_{i} \mid 1 \leq i \leq m\right\}$, with $x_{1}<x_{2}<\cdots<x_{m}$, be a period- $m$ orbit of $f$. Let $x_{s}=\max \{x \in P \mid x<f(x)\}$. Then $x_{s+1} \leq f\left(x_{s}\right)$ and $f\left(x_{s+1}\right) \leq x_{s}$, so $f$ has a fixed point $z$ in $\left[x_{s}, x_{s+1}\right]$. Since $m$ is odd, for some integer $t$ such that $1 \leq t \leq m-1$ and $t \neq s$ the points $f\left(x_{t}\right)$ and $f\left(x_{t+1}\right)$ lie on opposite sides of $z$. Thus $f\left(\left[x_{t}, x_{t+1}\right]\right) \supset\left[x_{s}, x_{s+1}\right]$. For simplicity, we assume that $x_{t}<x_{s}$. If $x_{s+1} \leq x_{t}$, the proof is similar. Let $q$ be the smallest positive integer such that $f^{q}\left(x_{s}\right) \leq x_{t}$. Then $2 \leq q \leq m-1$.

First assume that $m=3$. Without loss of generality, we assume that $f\left(x_{1}\right)=x_{2}, f\left(x_{2}\right)=x_{3}$, and $f\left(x_{3}\right)=x_{1}$. Let $J_{0}=\left[x_{1}, x_{2}\right]$ and $J_{1}=\left[x_{2}, x_{3}\right]$. For any $n \geq 2$, we can apply Lemma 4 to the cycle $J_{0} J_{1} J_{1} \cdots J_{1} J_{0}$ of length $n$ to obtain a period- $n$ point. Accordingly, if $f$ has a period-3 point, then $f$ has periodic points of all periods. Now assume that $m>3$. Since $q$ is the smallest positive integer such that $f^{q}\left(x_{s}\right) \leq x_{t}, x_{t+1} \leq f^{i}\left(x_{s}\right)$ whenever $1 \leq i \leq q-1$. If $x_{t+1} \leq f^{q-1}\left(x_{s}\right)<x_{s}$, Lemma 4 applies to the cycle

$$
\left[x_{t}, f^{q-1}\left(x_{s}\right)\right]\left[f^{q-1}\left(x_{s}\right), z\right]\left[f^{q-1}\left(x_{s}\right), z\right]\left[x_{t}, f^{q-1}\left(x_{s}\right)\right]
$$

and establishes the existence of a period-3 point of $f$. If $f^{q-1}\left(x_{s}\right)=x_{s+1}$, we can apply Lemma 4 to the cycle

$$
\left[z, x_{s+1}\right]\left[x_{t}, x_{t+1}\right]\left[x_{s}, x_{s+1}\right]\left[z, x_{s+1}\right]
$$

to obtain a period-3 point of $f$.

We proceed assuming that $x_{s+1}<f^{q-1}\left(x_{s}\right)$. If $k=\min \left\{1 \leq i \leq q-1 \mid f^{q-1}\left(x_{s}\right) \leq f^{i}\left(x_{s}\right)\right\}$, then $x_{t+1} \leq f^{k-1}\left(x_{s}\right)<f^{q-1}\left(x_{s}\right)$, so either $x_{s+1} \leq f^{k-1}\left(x_{s}\right)<f^{q-1}\left(x_{s}\right)$ or $x_{t+1} \leq f^{k-1}\left(x_{s}\right) \leq x_{s}$. If $x_{s+1} \leq f^{k-1}\left(x_{s}\right)<f^{q-1}\left(x_{s}\right)$, we can invoke Lemma 4 for the cycle

$$
\left[f^{k-1}\left(x_{s}\right), f^{q-1}\left(x_{s}\right)\right]\left[z, f^{k-1}\left(x_{s}\right)\right]\left[z, f^{k-1}\left(x_{s}\right)\right]\left[f^{k-1}\left(x_{s}\right), f^{q-1}\left(x_{s}\right)\right]
$$

to obtain a period-3 point of $f$. If $x_{t+1} \leq f^{k-1}\left(x_{s}\right) \leq x_{s}\left(<z<f^{q-1}\left(x_{s}\right)\right)$, we choose $u$ in $\left[x_{t}, x_{t+1}\right]$ such that $f(u)=z$, pick $w$ in $\left[z, f^{q-1}\left(x_{s}\right)\right]$ with $f(w)=u$, and let $v$ in $\left[f^{k-1}\left(x_{s}\right), z\right]$ be a point such that $f(v)=w$. By applying Lemma 4 to the cycle $[u, v][z, w][u, v]$ and, for every even integer $n \geq 4$, to the cycle

$$
[u, v]([z, w][v, z])^{\frac{n-2}{2}}[z, w][u, v]
$$

(here $([z, w][v, z])^{\frac{n-2}{2}}$ represents $(n-2) / 2$ copies of $\left.[z, w][v, z]\right)$ of length $n$, we conclude that $f$ has periodic points of all even periods. On the other hand, let $J_{i}=\left[z: f^{i}\left(x_{s}\right)\right]$ for $i=0,1, \cdots, q-1$, where $[a: b]$ denotes the closed interval with $a$ and $b$ as endpoints. For any $n \geq m+1$, we appeal to Lemma 4 to the cycle of length $n \quad J_{0} J_{1} \cdots J_{k-1} J_{q-1}\left[x_{t}, x_{t+1}\right] J \cdots J J_{0}$, where $J=\left[x_{s}, x_{s+1}\right]$, to confirm the existence of a period- $n$ point.

\section{A proof of Sharkovsky's theorem.}

We now combine (a), (b), (c), and Lemma 1 to prove Sharkovsky's theorem. 
Theorem 6 (Sharkovsky). Assume that $f: I \rightarrow I$ is a continuous map. If $f$ has a period-m point, then $f$ also has a period-n point precisely when $m \prec n$ in the Sharkovsky's ordering defined as in Section 1.

Proof. By (b) and (c), we have $3 \prec 5 \prec 7 \prec \cdots \prec 2 \cdot 3$. If $f$ has period- $(2 \cdot m)$ points with $m \geq 3$ and odd, then $f^{2}$ has period- $m$ points. By (b), $f^{2}$ has period- $(m+2)$ points, which by Lemma 1(2) implies that $f$ has either period- $(m+2)$ points or period- $(2 \cdot(m+2))$ points. If $f$ has period- $(m+2)$ points, then by (b) $f$ also has period- $(2 \cdot(m+2))$ points. In either case, $f$ has period- $(2 \cdot(m+2))$ points. On the other hand, by (c) $f^{2}$ has period- 6 points and hence, by Lemma $1(2), f$ has period- $\left(2^{2} \cdot 3\right)$ points. Now if $f$ has period- $\left(2^{k} \cdot m\right)$ points with $m \geq 3$ and odd and if $k \geq 2$, then by Lemma $1(1) f^{2^{k-1}}$ has period- $(2 \cdot m)$ points. It follows from what we have just proved that $f^{2^{k-1}}$ has period- $(2 \cdot(m+2))$ points and period- $\left(2^{2} \cdot 3\right)$ points. In view of Lemma $1(2), f$ has period- $\left(2^{k} \cdot(m+2)\right)$ points and period- $\left(2^{k+1} \cdot 3\right)$ points. Furthermore, because $f$ has period- $\left(2^{k} \cdot m\right)$ points, $f^{2^{k}}$ has period- $m$ points. By (b), $f^{2^{k}}$ has period- $2^{n}$ points as long as $2^{n}>m$, so by Lemma $1(2) f$ has period- $\left(2^{k+n}\right)$ points for all integers $n$ such that $2^{n}>m$. Finally, if $f$ has period- $2^{i}$ points for some integer $i \geq 2$, then $f^{2^{i-2}}$ has period- 4 points. As a result of (a), $f^{2^{i-2}}$ has period-2 points, ensuring that $f$ has period- $2^{i-1}$ points. This proves the sufficiency of Sharkovsky's theorem.

For the converse, it suffices to assume that $I=[0,1]$. Let $T(x)=1-|2 x-1|$ be the tent map on $I$. Then for any $k \geq 1$ the equation $T^{k}(x)=x$ has exactly $2^{k}$ distinct solutions in $I$. It follows that $T$ has finitely many period- $k$ orbits. Among these period- $k$ orbits, let $P_{k}$ be one with the smallest diameter $\max P_{k}-\min P_{k}$. For any $x$ in $I$, let $T_{k}(x)=\min P_{k}$ if $T(x) \leq \min P_{k}$, $T_{k}(x)=\max P_{k}$ if $T(x) \geq \max P_{k}$, and $T_{k}(x)=T(x)$ if $\min P_{k} \leq T(x) \leq \max P_{k}$. It is then easy to see that $T_{k}$ has exactly one period- $k$ orbit, i.e., $P_{k}$, and no period- $j$ orbit for any $j$ with $j \prec k$ in the Sharkovsky's ordering (see also [1], pp. 32-34]). Now let $Q_{3}$ be any period-3 orbit of $T$ of minimal diameter. Then $\left[\min Q_{3}, \max Q_{3}\right]$ contains finitely many period-6 orbits of $T$. If $Q_{6}$ is one of smallest diameter, then $\left[\min Q_{6}, \max Q_{6}\right]$ contains finitely many period-12 orbits of $T$. We choose one, say $Q_{12}$, of minimal diameter and continue the process inductively. Let $q_{0}=\sup \left\{\min Q_{2^{n \cdot 3}} \mid n \geq 0\right\}$ and $q_{1}=\inf \left\{\max Q_{2^{n} \cdot 3} \mid n \geq 0\right\}$. Let $T_{\infty}(x)=q_{0}$ if $T(x) \leq q_{0}, T_{\infty}(x)=q_{1}$ if $T(x) \geq q_{1}$, and $T_{\infty}(x)=T(x)$ if $q_{0} \leq T(x) \leq q_{1}$. Then it is easy to check that $T_{\infty}$ has periodic points of least period $2^{n}$ for each $n \geq 0$, but has no periodic points of any other periods. This establishes the other direction in Sharkovsky's theorem.

Remark. Our method can also be used to prove that if $f$ has a periodic point of odd period $m>1$, but no periodic points of odd period strictly between 1 and $m$ then any periodic orbit of odd period $m$ must be a Štefan orbit (cf. [4]).

Acknowledgments. I would like to thank M. Misiurewicz, A. N. Sharkovsky, and the referee for many constructive suggestions that led to improvements in this note.

\section{References}

[1] L. Alsedà, J. Llibre, and M. Misiurewicz, Combinatorial Dynamics and Entropy in Dimension One, 2nd ed., World Scientific, Singapore, 2000. 
[2] R. Barton and K. Burns, A simple special case of Sharkovskii's theorem, Amer. Math. Monthly 107 (2000) 932-933.

[3] L. S. Block and W. A. Coppel, Dynamics in One Dimension, Lecture Notes in Math. no. 1513, Springer-Verlag, Berlin, 1992.

[4] K. Burns, A note about Sharkovskii's theorem, preprint (2003).

[5] M. Misiurewicz, Remarks on Sharkovsky's theorem, Amer. Math. Monthly 104 (1997) 846-847.

[6] A. N. Sharkovsky, Coexistence of cycles of a continuous map of a line into itself, Ukrain. Mat. Zh. 16 (1964) 61-71 (Russian); English translation, Internat. J. Bifur. Chaos Appl. Sci. Engrg. 5 (1995) 1263-1273.

[7] P. Štefan, A theorem of Šarkovskii on the existence of periodic orbits of continuous endomorphisms of the real line, Comm. Math. Phys. 54 (1977) 237-248.

[8] P. D. Straffin, Jr., Periodic points of continuous functions, Math. Mag. 51 (1978) 99-105. 